\title{
Pregnancy Outcomes at Kasungu Maternity Ward in Central Malawi-A Review of Maternity Ward Register
}

\author{
Joo Heon Park1, Jin Sik Song1, James G. Kim¹, Changhyun Han¹, Diane J. Moon², Byungchan Kim³, \\ James Kachingwe ${ }^{4}$, George Talama ${ }^{4 *}$ \\ ${ }^{1}$ Youth with Talents, Fairfax, VA, USA \\ ${ }^{2}$ The Gwinnett School of Mathematics, Science, and Technology, Lawrenceville, GA, USA \\ ${ }^{3}$ Biomedical Engineering, Washington University, St. Louis, MO, USA \\ ${ }^{4}$ Kasungu District Hospital, Kasungu, Malawi \\ Email: *paulschung08@gmail.com
}

How to cite this paper: Park, J.H., Song, J.S., Kim, J.G., Han, C., Moon, D.J., Kim, B., Kachingwe, J. and Talama, G. (2019) Pregnancy Outcomes at Kasungu Maternity Ward in Central Malawi-A Review of Maternity Ward Register. Advances in Reproductive Sciences, 7, 51-59.

https://doi.org/10.4236/arsci.2019.73007

Received: January 31, 2019

Accepted: June 8, 2019

Published: June 11, 2019

Copyright (๑) 2019 by author(s) and Scientific Research Publishing Inc. This work is licensed under the Creative Commons Attribution International License (CC BY 4.0).

http://creativecommons.org/licenses/by/4.0/

\begin{abstract}
Health care services during pregnancy and childbirth and after delivery are important for the survival and wellbeing of both the mother and the infant. The pregnancy outcomes at Kasungu District Hospital Maternity Ward have not been documented. Additionally, MDHS does not capture data regarding, prematurity, APGAR scores, and causes of maternal deaths and causes of neonatal deaths. Using Kasungu District Hospital Maternity Ward register, we aimed to describe the pregnancy outcomes at Kasungu Maternity Ward. From March 2016 to February 2017, data were available for 10,842 deliveries. The calculated Perinatal Mortality Rate (PMR) was about 77/1000 births and the Maternal Mortality Ratio (MMR) was 318 deaths per 100,000 live births. The Spontaneous Vertex Delivery (SVD) rate was $86 \%$ and the caesarean section rate was $10 \% .1734(16 \%)$ of all deliveries were premature borne between 28 and 36 gestation weeks. 1182 (11\%) deliveries had missing APGAR scores and 81 neonates were born with 5 min Apgar scores less than 7. Adverse pregnancy outcomes occur at Kasungu Hospital Maternity Ward. More effort and resources are needed to decrease their occurrence.
\end{abstract}

\section{Keywords}

Pregnancy Outcomes, Mode of Delivery, Perinatal Deaths, Stillbirths, Maternal Deaths, APGAR Scores

\section{Background}

Health care services during pregnancy and childbirth and after delivery are im- 
portant for the survival and wellbeing of both the mother and the infant.

Maternal mortality ratio for Malawi is 439 per 100,000 live births and the under-5 mortality rate is 63 deaths per 1000 live births. The perinatal mortality rate is 35 deaths per 1000 pregnancies and the under-5 mortality declined from 234 deaths per 1000 live births in 1992 to 63 deaths per 1000 live births in 2015-16 [1].

In a study conducted to better understand the common and avoidable factors that affect perinatal mortality in Addis Ababa, Ethiopia, Getiye and Fantahun in 2017, it is found that birth interval less than 2 years, preterm delivery, anemia, congenital anomaly, previous history of early neonatal death and low birth weight were significantly associated with increased risk of perinatal mortality [2].

Access to caesarean section can reduce maternal and neonatal mortality and complications such as obstetric fistula.

The World Health Organization (WHO) advises that caesarean section should only be done when there is an obstetric indication, and does not recommend a target rate for countries to achieve at the population level [3].

The Apgar score system has continuing value for predicting neonatal and post-neonatal adverse outcomes interm as well as preterm infants.

Fei Li et al. in 2013 found that among births with a very low Apgar score at five minutes $(1-3)$, the neonatal and post-neonatal mortality rates remained high until term. On the other hand, among births with a high Apgar score (>7), neonatal and post-neonatal mortality rate decreased progressively with gestational age [4].

Stillbirths are currently not included in Millennium Development Goal tracking and remain invisible in global policies. Only about $2 \%$ of all stillbirths are counted through vital registration and global estimates are based on household surveys or modeling. $99 \%$ of stillbirths occur in low- and middle-income countries and one million stillbirths occur during birth [5].

Since 1992, the rates of C-sections have risen slightly. Only $3 \%$ of births occurred with C-section in 1992-2000 compared with 5\% in 2010 and 6\% in 2015-16 [1].

The pregnancy outcomes at Kasungu District Hospital Maternity Ward have not been documented. MDHS does not capture data regarding, prematurity, APGAR scores, causes of maternal deaths and stillbirths.

We therefore aimed to review the Kasungu District Hospital Maternity Ward register for the selected pregnancy outcomes.

\subsection{Main Objective}

To review the Pregnancy Outcomes at Kasungu District Hospital Maternity Ward from 2016 to 2017.

\subsection{Specific Objective}

To describe the Perinatal mortality, Maternal mortality, Stillbirths, Caesarean-sections, 5 min APGAR scores, Prematurity and Mode of delivery at Ka- 
sungu District Hospital Maternity Ward.

\section{Materials and Methods}

This retrospective study was conducted in June 2017 at Kasungu District hospital in the central region of Malawi

We used Kasungu District Hospital Maternity Ward register from March 2016 to February 2017 to manually extract our data.

From the register we specifically reviewed data concerning: Perinatal mortality, Maternal mortality, Stillbirths, Caesarean-sections, 5 min APGAR scores, Prematurity and Mode of delivery.

Data was entered into Microsoft excel and analyzed using the same software. All the tables, charts and graphs used to display the results of this review were created using Microsoft excel and Microsoft word.

\section{Results}

From March 2016 to February 2017 in Figure 1, data was available for 10,842 deliveries at Kasungu district hospital maternity ward. 10,742 (98\%) of these deliveries were singleton births and $270(2 \%)$ of these deliveries were multiple births.

\subsection{Premature Births}

$1734(16 \%)$ in Figure 2 of all deliveries were premature borne between 28 and 36 gestation weeks.

\subsection{Minute APGAR SCORES}

Data on 5 minute APGAR scores refer to Table 1 was available for 9660 (89\%) deliveries. There was no data for 1182 (11\%) deliveries on 5 minute APGAR

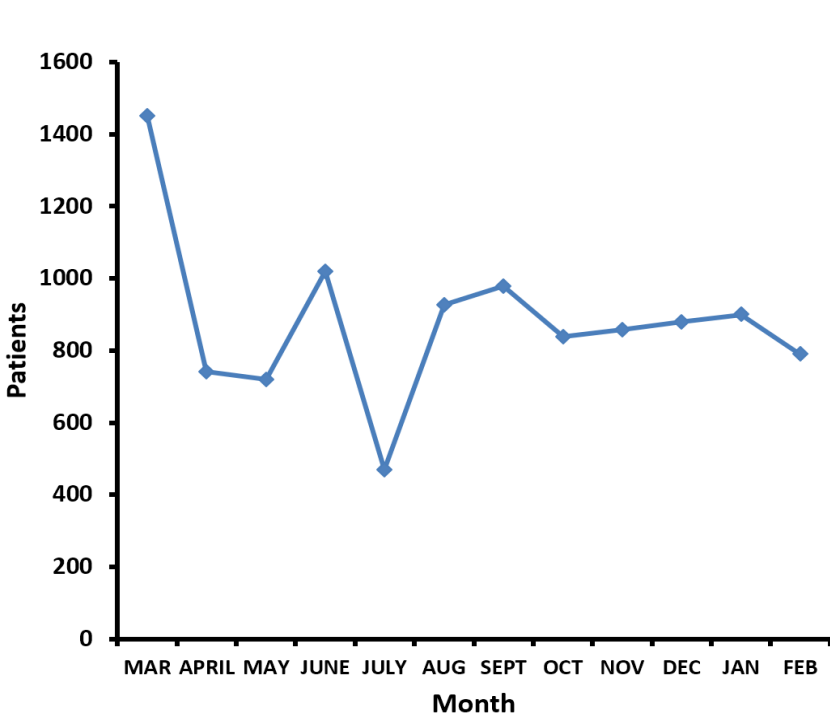

\begin{tabular}{|c|c|c|}
\hline MONTH & PATIENTS \\
\hline MAR & 1451 \\
\hline APRIL & 741 \\
\hline MAY & 720 \\
\hline JUNE & 1019 \\
\hline JULY & 470 \\
\hline AUG & 927 \\
\hline SEPT & 979 \\
\hline OCT & 838 \\
\hline NOV & 858 \\
\hline DEC & 879 \\
\hline JAN & 900 \\
\hline FEB & 790 \\
\hline TOTAL & 10572 \\
\hline
\end{tabular}

Figure 1. Deliveries over March 2016-February 2017. 




Figure 2. Premature births.

Table 1. Number of neonates born with 5 min APGAR scores either less than or greater than 7.

\begin{tabular}{cc}
\hline APGAR $(5 \mathrm{~min})$ & Count \\
\hline $7+$ & 9579 \\
$<7$ & 81 \\
Missing & 351 \\
\hline
\end{tabular}

scores (data was missing from the Register). 81 neonates were born with 5 min Apgar scores less than 7.

\subsection{Method of Delivery}

The most common method of delivery refer to Figure 3 was spontaneous vertex delivery (SVD) [9327 (86\%)], seconded by Caesarean section (CS) [1132 (10\%)], Vacuum extraction (VE) [180 (2\%)] and Breech extraction (BR) [203 (2\%)].

\subsection{Perinatal Deaths}

Over the period, there were 10,364 live-births and 478 (4.4\%) stillbirths. Of the stillbirths, 163 were fresh stillbirths and 315 were macerated stillbirths.

There was no neonatal death that occurred after the first week of life hence the neonatal mortality rate was not calculated.

There were 353 early neonatal deaths refer to Table 2 over the same period making a total of 831 peri-natal deaths giving the calculated perinatal mortality rate (PMR) of about $77 / 1000$ births.

The commonest cause of neonatal mortality was Birth Asphyxia (216) deaths in Table 3 followed by prematurity (109) and neonatal sepsis (18).

\subsection{Maternal Deaths}

During this period, there were 33 maternal deaths and a total of 10,364 live births giving a hospital specific maternal mortality ratio of 318 deaths/100,000 live births.

The commonest cause of maternal deaths in Table 4 was Obstetric Hemorrhage (16) followed by Eclampsia (6) and sepsis (4).

\section{Discussion}

Total monthly deliveries peaked in the month of July (1451) and dropped in the month of July (570). 


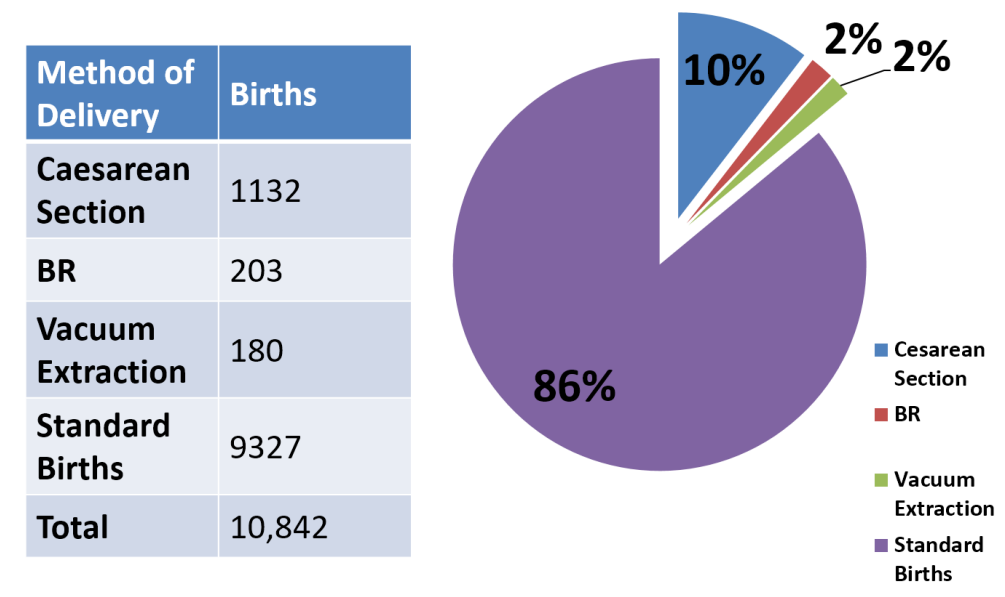

Figure 3. Method of delivery and births.

Table 2. Perinatal deaths.

\begin{tabular}{cc}
\hline Category & Count (\%) \\
\hline Total Deliveries & 10842 \\
Live Births & $10364(95.6 \%)$ \\
Still Births & $478(4.4 \%)$ \\
Fresh & $163(1.57 \%)$ \\
Macerated & $315(3.01 \%)$ \\
Neonatal Deaths & $353(3.26 \%)$ \\
Total Deaths & $831(7.66 \%)$ \\
\hline
\end{tabular}

Table 3. Neonatal deaths due to complications.

\begin{tabular}{cc}
\hline Leading Complication & Count (\%) \\
\hline Asphyxia & $216(61.2 \%)$ \\
Prematurity & $109(30.9 \%)$ \\
Sepsis & $18(5.1 \%)$ \\
Low Body Weight & $4(1.13 \%)$ \\
Other & $6(1.7 \%)$ \\
Total & 353 \\
\hline
\end{tabular}

This trend is seen in many obstetric units in Malawi and there is no obvious reason for this but can be explained by the fact that in Malawi month of July is the coldest month of the year and many couples are more likely to spend night together at home.

Furthermore, the period of June and July in Kasungu is when people are out of their fields relaxing at home and selling their agricultural produce.

These 2 factors result in more conceptions occurring in the month of July and then more deliveries occurring in the month of March. 
Table 4. Maternal deaths: Total deliveries were 10,842, Total live births were 10,364, Maternal Deaths were 33, MMR: 318/100,000.

\begin{tabular}{cc}
\hline Obstetric Complication & Count \\
\hline PPH & 6 \\
ECL & 6 \\
APH & 5 \\
Sepsis & 4 \\
Ruptured Uterus & 4 \\
Anemia & 1 \\
Chorioamnionitis & 1 \\
Hemorrhage & 1 \\
Other & 1 \\
None & 2 \\
Missing & 4 \\
Total Deaths & 33 \\
\hline
\end{tabular}

$1734(16 \%)$ of all deliveries were premature and $109(6.3 \%)$ of these premature births ended up into neonatal deaths.

Being born premature is strongly associated with Birth Asphyxia (AS at 5 min < 7) as described by Maria Svenvik et al. in 2015 [6].

Other risks of prematurity include Hypothermia, Hypoglycemia, Respiratory Distress Syndrome (RDS), Necrotizing Enterocolitis (NEC), Intraventricular Hemorrhage (IVH), Infections and Ventricular Leucomalacia.

The Apgar score is a useful and immediate tool used in the assessment of newborns. The factors that influence its final score may be related with labor, mother or infant itself.

Low 5 min apgar scores are associated with cerebral palsy and abnormal neurodevelopment.

In a study to determine the association between low apgar scores and mortality, neonatal encephalopathy and neurodevelopment, RonaldCholaetal (2016) found that very low 5 minute Apgar scores (0 - 3) were associated with 73.3\% neonatal mortality [7].

Very low Apgar scores were associated with significant encephalopathy $(83.3 \%)$ at 6 to 12 hours of age.

In our review, $81(0.78 \%)$ neonates were born with 5 min Apgar score of $<7$. However, this number could be bigger due to higher number (1182) of missing data on apgar scores.

Birth Asphyxia alone was responsible for 216 (61\%) of all neonatal deaths and this means that most of the deliveries with missing apgar scores were more likely to result in low Apgar scores and birth asphyxia.

In their retrospective hospital-based study, Neil Abdurashid Ibrahim et al. 
(2017) found that the important determinant factors for Birth Asphyxia were illiteracy, low level of education (primary education), vacuum delivery and Forceps delivery [8].

Access to caesarean section can reduce maternal and neonatal mortality and complications such as obstetric fistula. However, caesarean section without an obstetric indication can put women at risk of short and long-term health problems.

Since 1985, the international healthcare community has considered the ideal rate for caesarean sections to be between $10 \%$ and $15 \%$ [3].

In our review, the caesarean section rate was $10 \%$ against the national prevalence of $6 \%$ and the World Health Organization (WHO) does not recommend a target rate for countries to achieve at the population level [1].

The caesarean section rate found in this review is above the national average by $4 \%$ and the value observed is not in agreement with what was found by MDHS2016 that the caesarean delivery rate is higher in urban than rural areas (12\% versus $5 \%)$.

This trend is due tothe fact that health facility rates of caesarean births vary widely depending on differences in the case mix of the obstetric populations they serve, in their capacity and provisions, and in clinical management protocols.

This means that population-based recommended caesarean section rate cannot be applied as the ideal rate at the hospital level.

The perinatal mortality rate encompasses both stillbirths and earlyneonatal deaths, and offers a better measure of the level of mortality and quality of service around delivery.

In our review, there were 478 stillbirths and total deliveries were 10,842, giving stillbirth rate of $4.4 \%$.

There were 353 early neonatal deaths and the calculated perinatal mortality rate (PMR) was about 77 deaths/1000 births.

The observed perinatal mortality rate (77 deaths per 1000 births) is higher than the national average of 35 deaths per 1000 births and higher than that observed by MDHS 2016 in urban areas(40 deaths per 100 births) and in rural areas (34 deaths per 1000 births).

During this period, there were 33 maternal deaths and a total of 10,364 live births giving a hospital specific maternal mortality ratio (MMR) of 318 deaths/100,000 live births lower than the national average of 439 maternal deaths per 100,000 live births [1].

In a systematic analysis by the UN Maternal Mortality Estimation Inter-Agency Group, Leontine Alkema et al. (2016) found a MMR in Sub-Saharan Africa of 546 deaths/100,000 live births. In the same analysis, the global maternal mortality ratio fell from 385 deaths per 100,000 live births in 1990 to 216 deaths per 100,000 live births in 2015 representing a decline of $43 \cdot 9 \%$ [9].

MDHS 2010 reported MMR of 675 deaths per 100,000 live births while MDHS 2015-16 reported MMR of 497 deaths per 100,000 live births representing a decline in MMR of $26.4 \%$ [1]. 
Data for the causes of maternal deaths are needed to inform policies to improve maternal health. However, it was noted that MDHS 2015-16 did not report on the causes of maternal deaths.

Lale Say et al. in 2014 conducted a systematic analysis on global causes of maternal deaths and found that the three main global causes of maternal deaths were; Hemorrhage (27.1\%), hypertensive disorders $(14.0 \%)$ and sepsis $(10.7 \%)$ [10].

This trend was also noted in our review, and the three main causes of maternal deaths during this review were Obstetric Hemorrhage (16 deaths) followed by hypertensive disorders (6 deaths) and sepsis (4 deaths).

\section{Conclusion}

Adverse pregnancy outcomes occur at Kasungu District Hospital Maternity Ward. More resources and effort are needed to decrease their occurrence. Birth attendants should improve on Apgar scoring of neonates to avoid missing data in the register. Causes and events surrounding the stillbirths should be well documented in the register.

\section{Acknowledgements}

The authors acknowledge the Hememics Biotechnology, Inc and Youth with Talents for sponsoring this medical project. (Project \#: HB2017-06PH). We would like to thank staff from Kasungu District Hospital for their advice and assistance throughout this study. Furthermore, we are thankful for the permission and opportunity to conduct a retrospective analysis on their hospital data.

\section{Conflicts of Interests}

None declared.

\section{References}

[1] National Statistical Office (2015-2016) Maternal and Child Health Section. Malawi Demographic and Health Survey.

[2] Getiye, Y. and Fantahun, M. (2017) Factors Associated with Perinatal Mortality among Public Health Deliveries in Addis Ababa. BMC Pregnancy Childbirth, 17, 245. https://doi.org/10.1186/s12884-017-1420-7

[3] World Health Organization (2015) Human Reproduction Programme-Research for Impact.

[4] Li, F., Wu, T., Lei, X., Zhang, H., Mao, M., et al. (2013) The Apgar Score and Infant Mortality. PLoS ONE, 8, e69072. https://doi.org/10.1371/journal.pone.0069072

[5] Lawn, J.E., et al. (2010) Global Report on Preterm Birth and Stillbirth (1 of 7): Definitions, Description of the Burden and Opportunities to Improve Data. BMC Pregnancy and Childbirth, 10, S1. http://www.biomedcentral.com/1471-2393/10/S1/S1 https://doi.org/10.1186/1471-2393-10-s1-s1

[6] Svenvik, M., Brudin, L. and Blomberg, M. (2015) Preterm Birth: A Prominent Risk Factor for Low Apgar Scores. BioMed Research International, 2015, Article ID: 978079. https://doi.org/10.1155/2015/978079 
[7] Chola, R., et al. (2016) The Association between 5 Minute APGAR Score in Term Newborns and Mortality, Neonatal Encephalopathy and Neurodevelopment at 8 Weeks Postnatal age at the University Teaching Hospital in Zambia.

[8] Ibrahim, N.A., Mahye, A. and Abdulie, S. (2017) Prevalence of Birth Asphyxia and Associated Factors among Neonates Delivered in Dilchora Referral Hospital, in Dire Dawa, Eastern Ethiopia. Clinics in Mother Child Health, 14, 279. https://doi.org/10.4172/2090-7214.1000279

[9] Alkema, L., et al. (2016) Global, Regional, and National Levels and Trends in Maternal Mortality between 1990 and 2015, with scenario-Based Projections to 2030: A Systematic Analysis by the UN Maternal Mortality Estimation Inter-Agency Group. Lancet, 387, 462-467. https://doi.org/10.1016/s0140-6736(15)00838-7

[10] Say, L., et al, (2014) Global Causes of Maternal Death: A WHO Systematic Analysis. Lancet Global Health, 2, e323-e333. 